\title{
Neutron spectroscopy by thermalization light yield measurement in a composite heterogeneous scintillator
}

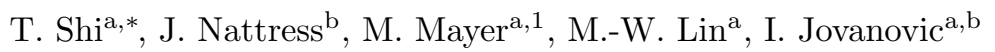 \\ ${ }^{a}$ Department of Mechanical and Nuclear Engineering, Pennsylvania State University, \\ University Park, PA 16802, USA \\ ${ }^{b}$ Department of Nuclear Engineering and Radiological Sciences, University of Michigan, \\ Ann Arbor, MI 48109, USA
}

\begin{abstract}
An exothermic neutron capture reaction can be used to uniquely identify neutrons in particle detectors. With the use of a capture-gated coincidence technique, the sequence of scatter events that lead to neutron thermalization prior to the neutron capture can also be used to measure neutron energy. We report on the measurement of thermalization light yield via a time-of-flight technique in a polyvinyl toluene-based scintillator EJ-290 within a heterogeneous composite detector that also includes ${ }^{6} \mathrm{Li}$-doped glass scintillator. The thermalization light output exhibits a strong correlation with neutron energy because of the preference for near-complete energy deposition prior to the ${ }^{6} \mathrm{Li}(\mathrm{n}, \mathrm{t})^{4} \mathrm{He}$ neutron capture reaction. The nonproportionality of the light yield from nuclear recoils contributes to the observed broadening of the distribution of thermalization light output. The nonproportional dependence of the scintillation light output in the EJ-290 scintillator as a function of proton recoil energy has been characterized in the range of $0.3-14.1 \mathrm{MeV}$ via the Birks parametrization through a combination of time-of-flight measurement and previously conducted measurements with monoenergetic neutron sources.
\end{abstract}

Keywords: Capture-gated detection, Fast neutron spectroscopy, ${ }^{6}$ Li-doped scintillator, EJ-290 scintillator, Light yield measurement

\footnotetext{
* Corresponding author.

Email address: tan.shi0122@gmail.com (T. Shi)

${ }^{1}$ Also at Pacific Northwest National Laboratory, Richland, WA 99354, USA
}

Preprint submitted to Nuclear Instruments and Methods in Physics Research Section ASeptember 18, 2016

C) 2016. This manuscript version is made available under the Elsevier user license http://www.elsevier.com/open-access/userlicense/1.0/ 


\section{Introduction}

Capture-gated neutron detection has shown great promise in neutron spectroscopy [1-4] due to the quasi-full energy deposition prior to neutron capture and the resulting reduced continuum in the detector response function. The capability to perform both thermal neutron detection and fast neutron spectroscopy in a single detector makes the capture-gated detection attractive in various applications. Here, we report on the neutron spectroscopic measurement of a recently developed heterogeneous composite scintillator that includes polyvinyl toluene (PVT)-based scintillator EJ-290 and ${ }^{6} \mathrm{Li}$-doped scintillating glass (GS20) rods. The composite detector exhibits excellent gamma/neutron discrimination capabilities due to the large Q-value of the neutron capture reaction ${ }^{6} \mathrm{Li}(\mathrm{n}, \mathrm{t})^{4} \mathrm{He}$ and has shown the advantage of low gamma misclassification rate for neutron detection in abundant gamma background, such as detection of delayed neutron emission from fissionable materials [5]. Fast neutron spectroscopy can benefit such applications because the neutron energy information provides insights about the nature of the neutron source. In this work, the neutron time-of-flight (TOF) was used to determine the neutron energy and, in conjunction with the capture-gated technique, the correlation between the light output of neutron thermalization pulse and the incident neutron energy was investigated. The scintillating polymer EJ-290 [6, 7] and its equivalents BC490 and NE-120 have already been used for constructing capture-gated detector prototypes and find their applications in radiation dosimetry [8] and nuclear safeguards [9]. However, the light yield nonproportionality of EJ-290 scintillator has not been measured. Here we describe the characterization of proton recoil light yield nonproportionality of the EJ-290 scintillator by use of spectroscopic capture-gated measurements in a composite heterogeneous detector based on EJ-290 scintillator and ${ }^{6}$ Li-doped glass (GS20). Spectroscopic measurements are aided with Monte Carlo simulation of detector response, which allows the EJ-290 nonproportionality to be parametrized using the Birks model. 
A detailed description of the detector geometry optimization, prototype fabrication, and performance characterization can be found in Refs. [7, 10]. The composite detector exhibits pulse shape discrimination (PSD) properties due to the different scintillation decay times of the two distinct materials used in the composite. The EJ-290 scintillator was selected as the neutron moderation material for two major reasons: first, the light emission and absorption spectra of the EJ-290 scintillator and GS20 glass are compatible and allow light transmission; second, the EJ-290 casting resin is relatively flexible for fabrication because it can be mixed with other materials in the form of scintillator solvent and cured. When a neutron is captured in the composite detector, two scintillation pulses are usually generated. Prior to the neutron capture reaction ${ }^{6} \mathrm{Li}(\mathrm{n}, \mathrm{t}){ }^{4} \mathrm{He}$, the neutron is thermalized in the PVT component of the scintillator, where multiple neutron scatters typically take place in rapid succession. The light output from those multiple scatters is detected using a photomultiplier tube as a single scintillation pulse, referred to as the thermalization pulse. The thermalization pulse precedes the neutron capture pulse, which is used to identify a neutron event. When a thermalization event is correlated to a capture event, the light output of the thermalization pulse is highly representative of the neutron energy on an event-by-event basis. This strong correlation can be understood by considering the dependence of the capture probability on the extent of neutron thermalization. As illustrated in Fig. 1, the results from a Geant 4 Monte Carlo simulation show that $75 \%$ of the neutrons lose more than $99 \%$ of their energy by proton and carbon recoils at the time of capture when the composite detector is irradiated with ${ }^{252} \mathrm{Cf}$ neutron source. Therefore, the thermalization pulse is a strong indicator of the incident neutron energy since the majority of the captured neutrons lose most of their energy to thermalization. It is noteworthy that, although the term "thermalization pulse" is used, not all of the captured neutrons are fully thermalized at the time of capture. A fraction of neutrons are captured at the time their energy is in the epithermal region or above. The ${ }^{6} \mathrm{Li}$ neutron capture cross section resonance centered at $240 \mathrm{keV}$ causes a fraction of neutrons to capture when their energy is in the 


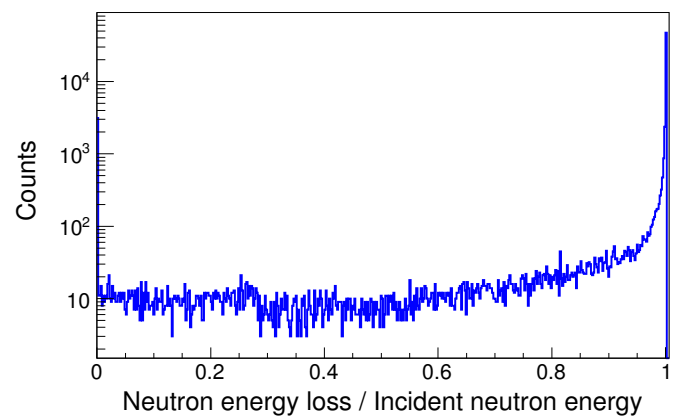

Figure 1: Simulated ratio of the neutron energy deposition at the time of capture to its incident energy in the EJ-290 scintillator for neutrons incident from a ${ }^{252} \mathrm{Cf}$ fission source. In the Geant4 simulation, the geometry and material composition of the fabricated composite scintillator were used [17]. A point source with ${ }^{252} \mathrm{Cf}$ fission spectrum was directed at the side of the detector. A total of 62948 captured neutron events are shown.

\section{Experiment}

${ }_{71}$ 2.1. Experimental setup and data analysis procedure

The relationship between the light output of the EJ-290 scintillator and the 


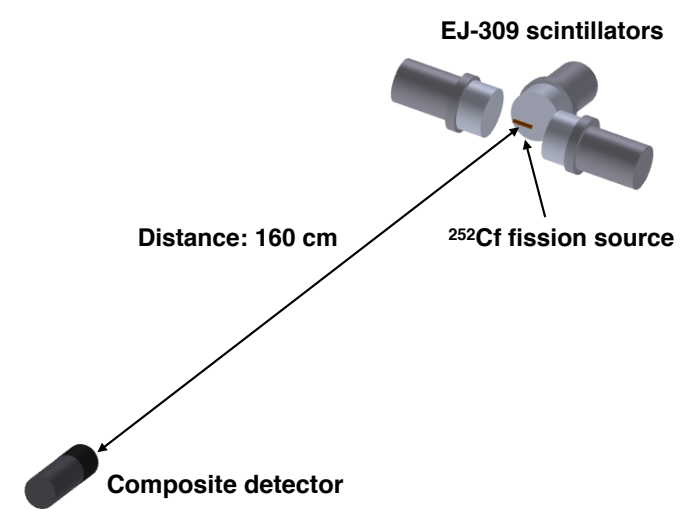

Figure 2: Experimental setup for the TOF measurement.

\begin{abstract}
in a number of earlier studies $[18,19]$. In this work, the neutron TOF measurement is used in conjunction with the capture-gated technique, which requires the time correlation of three events: gamma event to tag the fission event timing, neutron thermalization event, and neutron capture event. The TOF measurements were performed by tagging the fission events produced in a ${ }^{252} \mathrm{Cf}$ source with a spontaneous fission rate of $20.9 \mu \mathrm{Ci}$ over a period of 120 hours. Prompt neutrons and gamma rays are emitted nearly simultaneously in a fission event, so that the time of fission can be tagged using gamma rays, while the energy of neutrons detected in the composite detector can be determined from the neutron TOF. The experimental setup is depicted in Fig. 2. In the measurement, three calibrated EJ-309 liquid scintillation detectors were placed $5 \mathrm{~cm}$ away from the ${ }^{252} \mathrm{Cf}$ source. The composite detector was placed $1.6 \mathrm{~m}$ away from the source and $1 \mathrm{~m}$ above the concrete laboratory floor. A gamma event identified through PSD in any one of the three EJ-309 detectors was used as the initial signal of a fission event. Events within the TOF acceptance window of $50 \mathrm{~ns}<\mathrm{TOF}<410 \mathrm{~ns}$ were selected as neutron thermalization pulse candidates from the composite detector, corresponding to incident neutron energies $E_{n}$ of $5 \mathrm{MeV}>E_{n}>80 \mathrm{keV}$. The thermalization pulse cannot be distinguished solely on the basis of pulse shape, since both the gamma rays and neutrons interact with the EJ-290 scintil-
\end{abstract}


lator, which does not exhibit PSD. The thermalization pulse is therefore selected as the pulse preceding the neutron capture pulse within an estimated range of neutron diffusion time. The neutron capture pulses were identified through a two-dimensional criterion based on a fit of a pulse to a combination of standard pulse shape parameter (ratio of the pulse tail area to total pulse area) and the total area, as described in Ref. [7]. Only the events that lie within the $3 \sigma$ region of the neutron peak area were selected as neutron capture events. Simulations of the composite detector conducted using the Geant4 Monte Carlo code [20] reveal the mean neutron thermalization-to-capture (diffusion) time of $2.67 \mu \mathrm{s}$, with $>99 \%$ of the capture events having a diffusion time $<50 \mu$ s [17]. Therefore, an inter-event timing gate of $50 \mu \mathrm{s}$, along with the pulse shape consistent with the event taking place in the EJ-290 scintillator, were used to select the thermalization events that precede the neutron capture pulses.

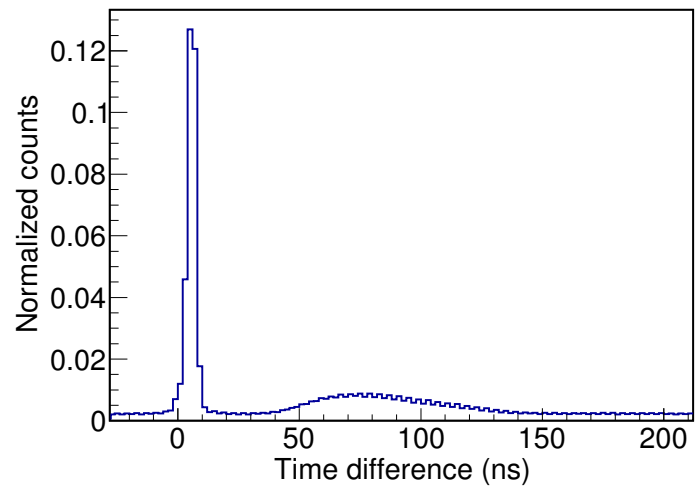

Figure 3: Normalized distribution of the inter-pulse delay between all triggered events in the composite detector and the gamma events from an EJ-309 liquid scintillation detector after the time delay correction in the TOF measurement. The peak centered at $\sim 5 \mathrm{~ns}$ is attributed to the prompt gamma arrival time difference between the two detectors due to the different distances of travel of two gamma rays. The peak centered at $\sim 75 \mathrm{~ns}$ is attributed to the difference in arrival time of a neutron in the composite detector and a gamma event in the EJ-309 detector. The FWHM of the DAQ time resolution is $4.8 \mathrm{~ns}$ based on the prompt gamma coincidence peak. 


\subsection{Resolution and calibration of the data acquisition system}

The digitizer used for data discretization (CAEN DT5730) has a sampling interval of 2 ns. The time delay among different digitizer channels was determined and corrected for by placing all detectors at equal distances from a ${ }^{60} \mathrm{Co}$ gamma source and using the two gamma rays that are emitted simultaneously following the ${ }^{60}$ Co beta decay. The data acquisition system (DAQ), including the photomultiplier rise time, had an overall time resolution of $4.8 \mathrm{~ns}$, measured at full-width-half-maximum of the inter-pulse delay distribution, which is shown in Fig. 3. The uncertainty of neutron energy increases with neutron energy due to the decrease of TOF. For a $2.1 \mathrm{MeV}$ neutron, which is the upper bound chosen for the subsequent analysis of the thermalization output spectra, the standard error induced by DAQ time resolution is $0.11 \mathrm{MeV}$. The increase of TOF path could further improve the TOF time resolution, but would also decrease the detector extrinsic efficiency. The uncertainty of the TOF path is limited by the thickness of the detector $(5.08 \mathrm{~cm})$ and finite thickness of the ${ }^{252} \mathrm{Cf}$ source $(\sim 0.5 \mathrm{~cm})$ that is encapsulated in the source package.

The electron equivalent light output from the composite detector was calibrated by the Compton edge of ${ }^{137} \mathrm{Cs}(0.477 \mathrm{MeV}),{ }^{60} \mathrm{Co}(0.963 \mathrm{MeV}), \mathrm{AmBe}$ $(4.20 \mathrm{MeV})$, and the double-escape peak of the $15.1 \mathrm{MeV}$ gamma ray from ${ }^{12} \mathrm{C}(14.078 \mathrm{MeV})$. The Compton edge location of the calibration source was validated by Geant4 simulation with the use of energy dependent resolution function via the method described in Ref. [21]. The thermalization pulse light output was calibrated to electron equivalent energy using the total pulse area. The finite voltage gain shift over time was also taken into account since the data were collected over multiple days. The DAQ was triggered by events occurring in the composite detector, using a pretrigger record width of $\sim 550$ ns to identify an immediately preceding fission signal. A six-hour-long neutron shadow cone experiment was also performed using the method described in Refs. [7] and [22] to account for the contribution of the neutron room scatter. It was determined from the shadow cone measurements that $\sim 13 \%$ of all events that were selected by the described criteria arise from neutron arriving indirectly, 
i.e. by undergoing room scatter prior to entering the detector.

\section{Results}

\subsection{Thermalization light output spectra from TOF measurement}
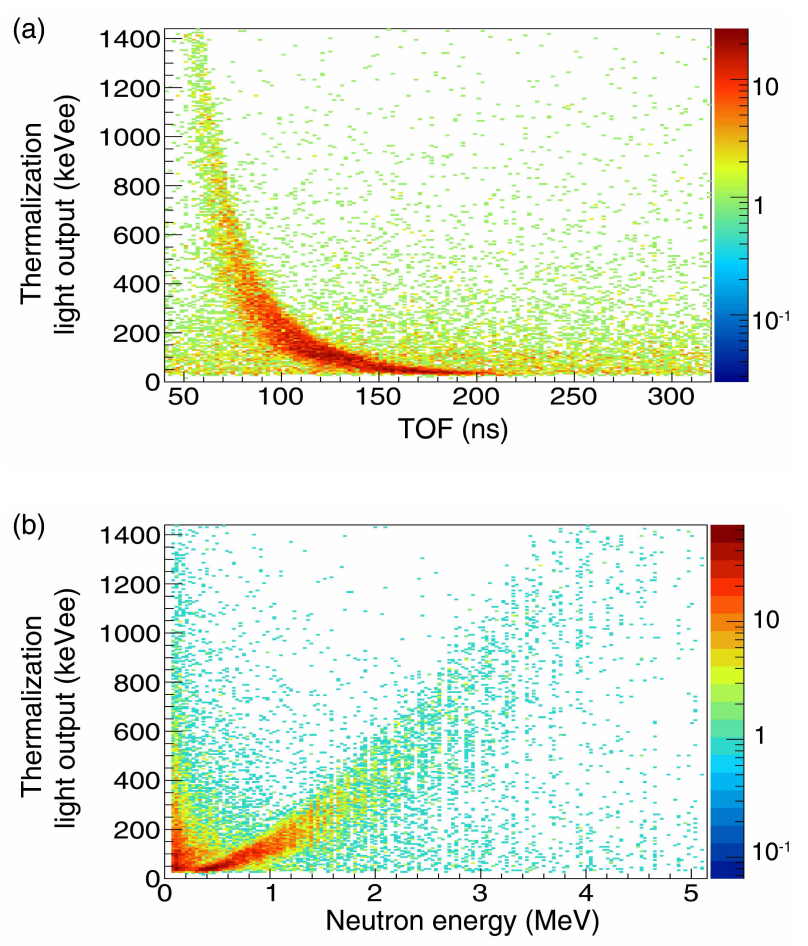

Figure 4: Relationship between (a) thermalization light output and TOF and (b) thermalization light output and neutron energy calculated from the TOF. The thermalization light output forms a band showing a clear nonlinear correlation with neutron energy. Events are largely concentrated at neutron energies $\lesssim 2 \mathrm{MeV}$ due to a combination of the shape of the neutron fission spectrum of ${ }^{252} \mathrm{Cf}$ and the reduction of detector efficiency with increasing neutron energy. At low neutron energies $(\lesssim 0.3 \mathrm{MeV})$, the room scatter and accidental events become significant, as evident from the vertical band at low energies. At higher energies, the vertical banding of the events is due to the discrete digitizer sampling time (2 ns).

As can be seen in Fig. 4, the thermalization light output at any incident neutron energy shows a sharp maximum and clustering into a band. Although the energy deposition does not necessarily result in the same light output due to 
a variety of possible scattering sequences on hydrogen and carbon and the variations of the quenching factor, the shape of the distribution of light output still reflects the quasi-full energy deposition of the incident neutron in the EJ-290 scintillator. The characteristic range of light output results in a finite width of the light output band, which is readily discerned in Fig. 4. The maximum of the light output at any given neutron energy, observed as the sharp band cutoff, is attributed to a single neutron backscatter on hydrogen, in which the entire neutron energy is deposited in a single collision. At higher neutron energies, inelastic scattering and competing reactions such as ${ }^{12} \mathrm{C}(\mathrm{n}, \alpha){ }^{9} \mathrm{Be},{ }^{12} \mathrm{C}\left(\mathrm{n}, \mathrm{n}^{\prime}\right) 3 \alpha$ with a threshold of $6.17 \mathrm{MeV}$ and $7.98 \mathrm{MeV}$, respectively, could also complicate the detector response. The correlated thermalization-to-capture time extracted from the TOF measurement is consistent with Monte Carlo simulation corrected for the expected exponentially dependent accidental coincidence rate, as shown in Fig. 5. The obtained capture time distribution also confirms the suitability of the gating time chosen for the experiment.

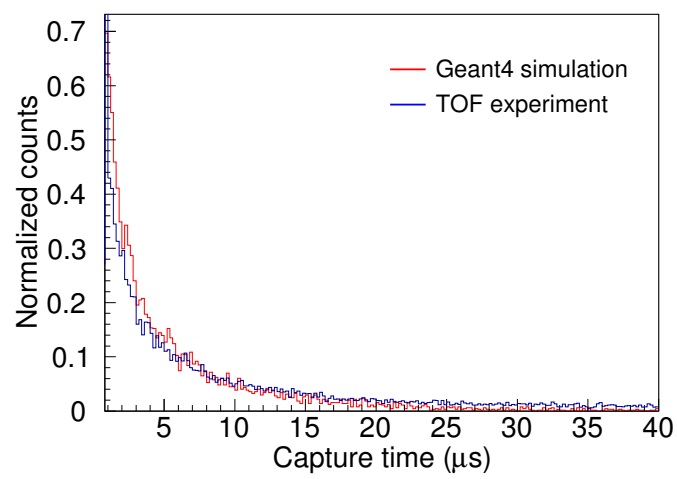

Figure 5: Measured and simulated thermalization-to-capture time with ${ }^{252} \mathrm{Cf}$ source normalized to the number of events detected from $0.8 \mu$ s to $40 \mu \mathrm{s}$. All measured events are correlated with the TOF of the incident neutron. The shortest measured capture time of $0.8 \mu$ s is limited by the waveform record length.

160 The thermalization pulse area spectra have been extracted in the range of 0.3 MeV-2.1 MeV with a bin size of $200 \mathrm{keV}$ and are shown in Fig. 6 . In the 
range of $0.3-1.1 \mathrm{MeV}$, the shape of the spectrum resembles a single peak. As the energy is increased, the peak is broadened and cannot be resolved as well due in part to the low efficiency of the composite detector at higher neutron energies. Such peak broadening has also been witnessed in a previous measurement that used a monoenergetic DD neutron source (2.45 MeV) [17]. As discussed earlier, this feature of the spectrum is a consequence of the variation of the scattering sequence prior to neutron capture. Nevertheless, the continuum extending to low light output is largely suppressed and a peak-like structure can be observed, unlike the spectra measured in non-capture-gated hydrogen-based organic scintillators in the same energy region [18]. The maximum light output corresponds to complete transfer in a single collision with a hydrogen nucleus, while multiple collisions with hydrogen and carbon nuclei generate less light output due to the nonlinear quenching factor of the nuclear recoils [3, 19, 23]. Furthermore, a fraction of the neutrons are captured before their kinetic energy is fully dissipated in collisions, which can also account for a fraction of the peak broadening. Finally, the spectral feature is also broadened by the intrinsic resolution of the detector electronics and the neutron energy resolution encountered in the TOF measurement.

\subsection{Dependence of light output on neutron energy}

To extract the nonproportional response of the detector, the peaks of the spectra in Fig. 6 have been fitted by a Gaussian distribution: $f(x)=$ $a \exp \left[-(x-b)^{2} /\left(2 c^{2}\right)\right]$. It is noted that, due to the presence of accidental coincidence events, the shape of the peak could be altered slightly, which would influence the determination of the exact peak position of the light output distribution. Due to multiple scatters on both hydrogen and carbon and the light output nonproportionality, the peak location of the Gaussian fit, which represents the most probable light output when a neutron with a given energy is captured in the composite detector, corresponds to a light output lower than that produced by a full energy transfer in a single scatter on hydrogen.

The measured dependence of the light output on neutron energy, represented 
(a)
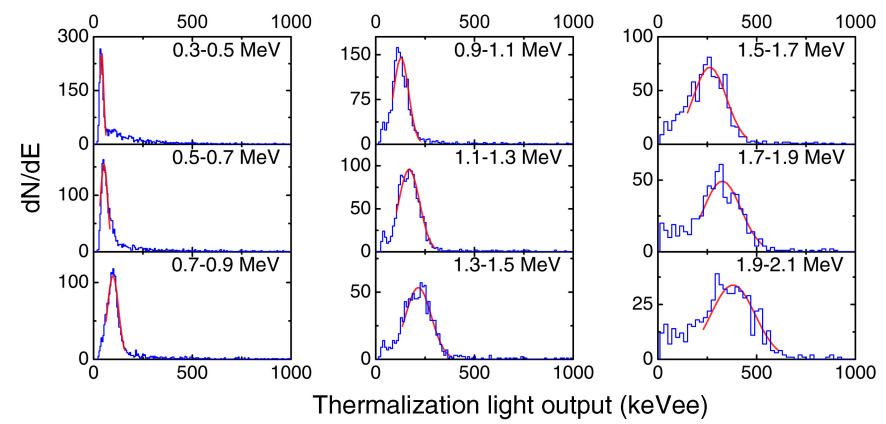

(b)
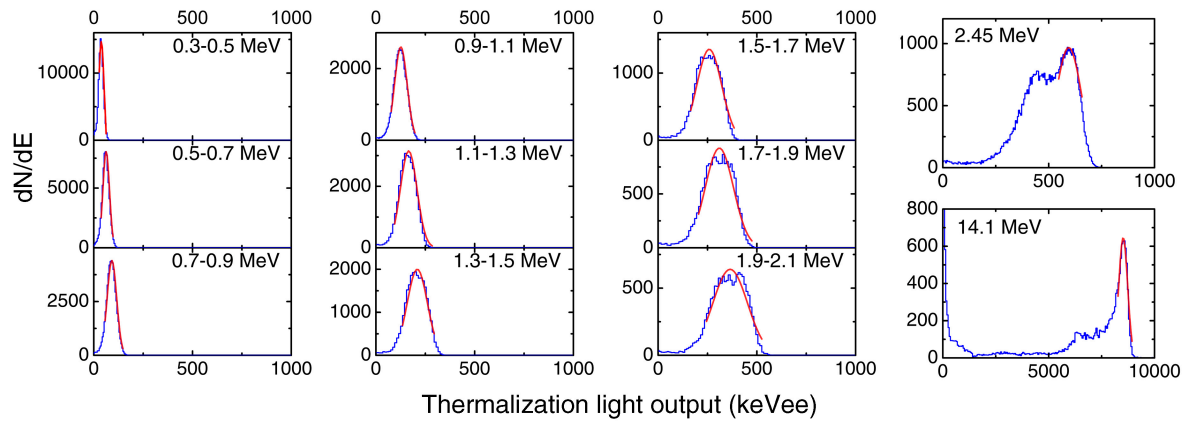

Figure 6: (a) Experimental thermalization pulse area spectra from TOF measurement with a neutron energy bin size of $200 \mathrm{keV}$. The thermalization light output is calibrated to the electron equivalent energy, where bin widths of $5 \mathrm{keVee}, 10 \mathrm{keVee}$, and $20 \mathrm{keVee}$ were applied for the 0.3-0.9 MeV, 0.9-1.5 MeV, and 1.5-2.1 MeV region, respectively, to account for statistical limitations at higher neutron energies. (b) Simulated thermalization pulse area spectra with Geant4 Monte Carlo code for the $0.3 \mathrm{MeV}-2.1 \mathrm{MeV}$ portion of the ${ }^{252} \mathrm{Cf}$ neutron fission spectrum, 2.45 MeV and $14.1 \mathrm{MeV}$ monoenergetic neutron source. The red curve represents a Gaussian fit to the high-energy part of the spectrum. 


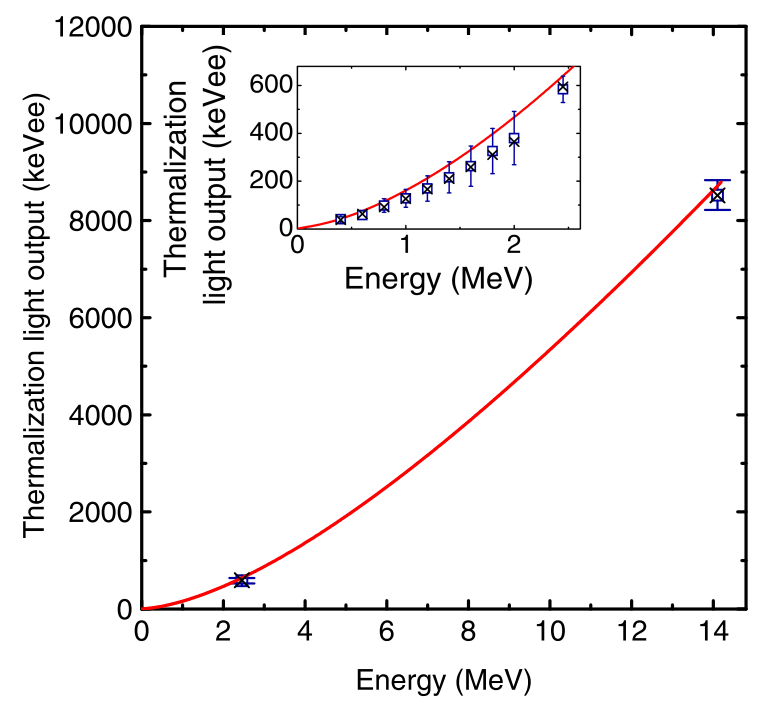

Figure 7: Light output nonproportionality curve (red curve) of proton recoil with Birks parametrization based on TOF and monoenergetic neutron data. Peak locations of experimental (square marker) and simulated (triangular marker) monoenergetic neutron light output are shown in the full-size image, while peak locations of experimental (square marker) and simulated (triangular marker) TOF data points and the $2.45 \mathrm{MeV}$ monoenergetic neutron data point are shown in the inset. The error bar is defined as one standard deviation of the Gaussian fit to the thermalization light output spectra. 
by the black square markers in Fig. 7, is nonlinear and mostly determined by the nonproportional light output from proton recoil in the EJ-290 scintillator. In order to obtain the proton light output function, an iterative method was used and aided by Geant4 simulation of the thermalization sequence to obtain the best fit to the peak locations of the light output spectra from the TOF measurement and previously conducted measurements with monoenergetic neutron sources (DD, 2.45 MeV and DT, 14.1 MeV) [17]. First, the proton light output function was parametrized via the Birks semi-empirical formula [12]. The Birks semi-empirical formula,

$$
L(E)=\int_{0}^{E} \frac{d L}{d x} d x=L_{0} \int_{0}^{E} \frac{d E}{1+k B d E / d x},
$$

has been successfully applied in explaining the experimental recoil nonproportionality in organic scintillators at both low energies $(<1 \mathrm{MeV})[24]$ and high energies (up to $20 \mathrm{AMeV}, \mathrm{AMeV}$ being a unit that describes energy-pernucleon) [25]. Here, $E$ is the initial particle energy, $L$ is the total light output, $-d E / d x$ is the stopping power, and $L_{0}$ and $k B$ are the fit parameters. According to Birks' theory, "damaged" molecules are produced locally along the track of the charged recoil and serve as the quenching agents for non-radiative recombination [12]. The term $k B(d E / d x)$ is a measure of the damaged molecule density that accounts for the energy-dependent quenching nonproportionality. In the analysis, the stopping power data of the PVT-based scintillator from the NIST pstar library were used [26]. The energy transferred from neutron to proton in each interaction can be extracted from the Geant4 simulation of the detector response. The energy transferred was then converted to the light output based on the Birks formula. Here, we ignored the light output from carbon recoils for two reasons: first, the average energy transferred to carbon per captured neutron is very small; second, the light yield of carbon is much less than that of a proton of the same energy [25]. The combination of these two effects leads to an overall negligible contribution of carbon recoils to the total light output for the neutron energy region studied [27]. The obtained proton light output in each interaction was then broadened by the detector energy resolution function. 
Next, the light output of all proton recoils produced from the same captured neutron was summed, corresponding to the experimental observable (total light output). The detector light output response to ${ }^{252} \mathrm{Cf}$ fission neutrons was simulated, where the light output spectra in the range of $0.3 \mathrm{MeV}-2.1 \mathrm{MeV}$ with a bin size of $200 \mathrm{keV}$ have been extracted so as to compare with the measurement spectra. Monoenergetic neutron sources $(2.45 \mathrm{MeV}$ and $14.1 \mathrm{MeV})$ were also simulated and all the simulated spectra were fitted with a Gaussian distribution, as shown in Fig. 6. Since the fit parameters $L_{0}$ and $k B$ are unknown, an iterative method was applied to simultaneously fit to the nine peak locations of the TOF measurement spectra shown in Fig. 6 and the peak locations of the 2.45 MeV and 14.1 MeV neutron light output spectra reported in Ref. [17]. The goodness of fit was measured via the Pearson's chi-squared test, where the $L_{0}$ and $k B$ were adjusted to find the minimum $\chi^{2}$ :

$$
\chi^{2}=\sum_{i=1}^{n}\left(O_{i}-E_{i}\right)^{2} / E_{i}
$$

Here, $E_{i}$ is the simulated peak location and $O_{i}$ is the experimental peak location. The parameter space search for the $\left(L_{0}, k B\right)$ pair was performed manually to seek the minimum $\chi^{2}$, where a fixed step size of $10 \mathrm{keVee} / \mathrm{MeV}$ for $L_{0}$ and a fixed step size of $0.1 \mathrm{~g} \mathrm{MeV}^{-1} \mathrm{~cm}^{-2}$ for $k B$ were used. A $\chi^{2}$ minimum exists in the parameter space, which corresponds to $L_{0}=1410 \pm 10 \mathrm{keVee} / \mathrm{MeV}$ and $k B=0.018 \pm 0.001 \mathrm{~g} \mathrm{MeV}^{-1} \mathrm{~cm}^{-2}$ with $\chi^{2}=2.9$ for 9 degrees of freedom. The error bar was defined as the size of the step employed in this parameter search. From Fig. 6, we can observe a good agreement between the experimental and simulated peak locations. They are located below the proton light output curve, which represents the maximum light output for a given neutron energy.

The nonproportionality of the EJ-290 PVT-based polymer and its equivalents has not been previously characterized in this manner. However, if compared with other PVT-based plastic scintillators, the same order of magnitude can be found for the fit parameters $k B$ and $L_{0}[28]$. We note that the material composition and the characteristics of the fabrication process (curing, degassing, etc.) can have a significant influence on the detector scintillation property. Ac- 
cording to Ref. [13], the chemical composition, purity, and previous exposure to radiation can all affect the detector response functions. For the same organic scintillator, it is difficult to obtain identical $k B$ value due to the difference of chemical composition, proton energy range studied, and the experimental approach applied [28], but the values should still be comparable. The obtained nonlinear fitting curve can be used to correlate the neutron energy from the thermalization pulse area spectrum for spectroscopic analysis. Nonproportionality of the light yield in organic scintillators is in general detrimental to energy resolution. Detector segmentation with separate light collection devices, as proposed [4] and realized [2], could potentially mitigate the broadening of the light output distribution and thus increase the energy resolution.

\subsection{Simulated thermalization light output spectra}

The simulated thermalization light output spectrum in response to incident neutrons from spontaneous fission of ${ }^{252} \mathrm{Cf}$ exhibits a peak at low energies (0.3 MeV-1.5 MeV). At higher energies (1.5 MeV-2.1 MeV), the spectrum exhibits a flatter peak response due to the greater variation of the scatter histories and proton light output nonproportionality. A general trend of broadening of the light output distribution with increasing neutron energy is observed. For 2.45 MeV and 14.1 MeV monoenergetic neutrons, the shoulder region on the low light output side of the main peak is observed both in the simulation and in the measurement [17]. If we assumed ideal proportionality in the proton light output, the shoulder region would be completely suppressed. With a neutron energy of $14.1 \mathrm{MeV}$, nuclear reactions involving ${ }^{12} \mathrm{C}$ need to be taken into account in the detector response, which is important in accurately simulating the low energy end of the spectrum. The simulation can also help understand the distribution of accidental events in the TOF measurement, which are mostly located at low neutron energies and low light output. An ideal shape of the light output spectrum would be a single, narrow peak for monoenergetic neutrons. From the simulation, we can study the impact of nonproportionality on the light output broadening, which will be useful for the future design of a neutron 
spectrometer.

\section{Conclusion}

Based on the spectroscopic characterization presented here and Ref. [17], a strong dependence between the neutron energy and the thermalization light output is observed in the composite detector, suggesting a potential for multiple functions in one single detector, including reliable neutron counting and neutron spectroscopy. Although the EJ-290 scintillator employed in the composite detector does not exhibit PSD capability, future detector design using moderation material with PSD capability will be beneficial for rejection of accidental coincidences of gammas with neutron captures, leading to more accurate neutron spectroscopy. It will also allow the detector to be used in both non-gated and capture-gated modes. Spectroscopic characterization with a ${ }^{252} \mathrm{Cf}$ source relies on a simple laboratory setup. However, the employment of TOF technique combined with capture-gated detection requires the time correlation of three events, which would significantly reduce the counting statistics per unit time. The choice of TOF path distance needs to be prudent because it determines both the detector extrinsic efficiency and the neutron energy resolution. Electronics with better time resolution is also desired for achieving an improved time resolution. Previous spectroscopic characterizations of capture-gated detector were mainly based on monoenergetic neutron source measurements $[16,29,30]$. TOF measurement with a ${ }^{252} \mathrm{Cf}$ source can provide a continuous response functions, but with a moderate energy resolution. In order to improve the energy resolution of the response functions, accelerator-based reactions can be used to produce continuous spectrum of neutrons with a wide range of neutron energies and produce a pulsed, directional neutron flux [19] for spectral characterization. For accurate neutron spectroscopic measurements without neutron unfolding, multiple detectors usually need to be utilized, such as the case of a proton recoil telescope, where two detectors and a collimated neutron source are 
often required $[31,32]$. While the recently developed ${ }^{6} \mathrm{Li}$-doped heterogeneous composite detector used in this work can be used to perform neutron spectral unfolding from an aggregate spectrum of many events, the peak-like structure in the capture gated thermalization light spectrum makes event-by-event energy reconstruction plausible. This characteristic is appealing for fast real-time spectral reconstruction in radiation dosimetry and nuclear security applications.

\section{Acknowledgements}

This research was supported by the National Science Foundation under Grant No. ECCS-1348366 and by the U.S. Department of Homeland Security under Grant Award Numbers 2014-DN-077-ARI078-02 and 2015-DN-077ARI096.

[1] J. B. Czirr, G. L. Jensen, Nucl. Instrum. Methods Phys. Res., Sect. A 284 (1989) 365-369.

[2] W. Feldman, G. Auchampaugh, R. Byrd, Nucl. Instrum. Methods Phys. Res., Sect. A 306 (1991) 350-365.

[3] T. Aoyama, K. Honda, C. Mori, K. Kudo, N. Takeda, Nucl. Instrum. Methods Phys. Res., Sect. A 333 (1993) 492-501.

[4] J. Abdurashitov, V. Gavrin, A. Kalikhov, V. Matushko, A. Shikhin, V. Yants, O. Zaborskaia, J. Adams, J. Nico, A. Thompson, Nucl. Instrum. Methods Phys. Res., Sect. A 476 (2002) 318-321.

[5] P. Rose, A. Erickson, M. Mayer, J. Nattress, I. Jovanovic, Sci. Rep. 6 (2016).

[6] G. Rich, K. Kazkaz, H. Martinez, T. Gushue, Nucl. Instrum. Methods Phys. Res., Sect. A 794 (2015) 15-24. 
[7] M. Mayer, J. Nattress, V. Kukharev, A. Foster, A. Meddeb, C. Trivelpiece, Z. Ounaies, I. Jovanovic, Nucl. Instrum. Methods Phys. Res., Sect. A 785 (2015) 117-122.

[8] D. Lewis, N. Spyrou, A. Williams, P. Beeley, Radiat. Prot. Dosim. 126 (2007) 390-393.

[9] M. Flaska, S. Pozzi, B. Czirr, et al., Use of an LGB detector in nuclear nonproliferation applications, in: Proc. IEEE Nucl. Sci. Symp. Conf. Rec., IEEE, 2008, pp. 3376-3380.

[10] M. Mayer, J. Nattress, C. Trivelpiece, I. Jovanovic, Nucl. Instrum. Methods Phys. Res., Sect. A 784 (2015) 168-171.

[11] A. Santamarina, D. Bernard, P. Blaise, M. Coste, A. Courcelle, T. Huynh, C. Jouanne, P. Leconte, O. Litaize, S. Mengelle, et al., JEFF report 22 (2009) 2 .

[12] J. B. Birks, P. Phys. Soc Lond. A 64 (1951) 874.

[13] R. Craun, D. Smith, Nucl. Instrum. Methods 80 (1970) 239-244.

[14] R. Breukers, C. Bartle, A. Edgar, Nucl. Instrum. Methods Phys. Res., Sect. A 701 (2013) 58-61.

[15] B. Fisher, J. Abdurashitov, K. Coakley, V. Gavrin, D. Gilliam, J. Nico, A. Shikhin, A. Thompson, D. Vecchia, V. Yants, Nucl. Instrum. Methods Phys. Res., Sect. A 646 (2011) 126-134.

[16] C. Bass, E. Beise, H. Breuer, C. Heimbach, T. Langford, J. Nico, Appl. Radiat. Isotopes 77 (2013) 130-138.

[17] J. Nattress, M. Mayer, A. Foster, A. Meddeb, C. Trivelpiece, Z. Ounaies, I. Jovanovic, IEEE Trans. Nucl. Sci 63 (2016) 1-9.

[18] N. Kornilov, I. Fabry, S. Oberstedt, F.-J. Hambsch, Nucl. Instrum. Methods Phys. Res., Sect. A 599 (2009) 226-233. 
[19] A. Enqvist, C. C. Lawrence, B. M. Wieger, S. A. Pozzi, T. N. Massey, Nucl. Instrum. Methods Phys. Res., Sect. A 715 (2013) 79-86.

[20] S. Agostinelli, J. Allison, K. a. Amako, J. Apostolakis, H. Araujo, P. Arce, M. Asai, D. Axen, S. Banerjee, G. Barrand, et al., Nucl. Instrum. Methods Phys. Res., Sect. A 506 (2003) 250-303.

[21] E. R. Siciliano, J. H. Ely, R. T. Kouzes, J. E. Schweppe, D. M. Strachan, S. T. Yokuda, Nucl. Instrum. Methods Phys. Res., Sect. A 594 (2008) 232-243.

[22] C. Eisenhauer, J. Hunt, R. Schwartz, Radiat. Prot. Dosim. 10 (1985) 43-57.

[23] A. Enqvist, I. Pázsit, Nucl. Instrum. Methods Phys. Res., Sect. A 618 (2010) 266-274.

[24] L. Reichhart, D. Y. Akimov, H. Araújo, E. Barnes, V. Belov, A. Burenkov, V. Chepel, A. Currie, L. DeViveiros, B. Edwards, et al., Phys. Rev. C 85 (2012) 065801.

[25] S. Nyibule, J. Tõke, E. Henry, W. Schröder, L. Acosta, L. Auditore, G. Cardella, E. De Filippo, L. Francalanza, S. Gianì, et al., Nucl. Instrum. Methods Phys. Res., Sect. A 768 (2014) 141-145.

[26] M. Berger, J. Coursey, M. Zucker, J. Chang, NIST Standard Reference Database 124 (2015). Available online at: http://www.nist.gov/pml/data/star/index.cfm.

[27] S. A. Pozzi, R. Oberer, J. S. Neal, IEEE Trans. Nucl. Sci 52 (2005) 31413146.

[28] L. Torrisi, Nucl. Instrum. Methods Phys. Res., Sect. B 170 (2000) 523-530.

[29] J. B. Czirr, G. L. Jensen, Nucl. Instrum. Methods Phys. Res., Sect. A 349 (1994) 532-539. 
385

386

387

388

389

390

30] E. Kamykowski, Nucl. Instrum. Methods Phys. Res., Sect. A 317 (1992) $559-566$.

[31] G. F. Knoll, Radiation detection and measurement, John Wiley \& Sons, 2010.

[32] M. Cambiaghi, F. Fossati, T. Pinelli, Nucl. Instrum. Methods 82 (1970) 106-108. 